\title{
SiRNA-Mediated IncRNA ANRIL Knockdown Enhances the Sensitivity of Gastric Cancer Cells to Doxorubicin
}

\author{
Jiahai Chen ${ }^{1}$, Xuejiang Dai ${ }^{1}$, Hebin Yu ${ }^{1}$, Fei Peng ${ }^{1}$ and Lina Chen ${ }^{1, *}$ \\ 1 Gastroenterology, Zhongxian Hospital People's Hospital of Chongqing, Jiankang Road, Chongqing, China
}

* Corresponding author: Lina Chen, Gastroenterology, Zhongxian Hospital People's Hospital of Chongqing, Jiankang Road, Chongqing, China. Tel: +8602354233080; Email: yyooonn@sina.com

Received 2020 August 21; Revised 2020 September 18; Accepted 2020 October 11.

\begin{abstract}
Background: Long non-coding RNAs (lncRNAs) play pivotal roles in carcinogenesis and the development of drug resistance in various malignancies.

Objectives: The current study aimed to explore the impact of antisense non-coding RNA in the INK4 locus (ANRIL) silencing on proliferation and the sensitivity of KATO III gastric cells to the doxorubicin as a common chemotherapeutic agent.

Methods: The KATO III cells were transfected with ANRIL siRNA (si-ANRIL) using Lipofectamine ${ }^{\mathrm{TM}} 2000$ reagent. Following that, the relative ANRIL levels were determined by quantitative Real-Time PCR. Trypan blue assay was conducted to observe the tumor cell proliferation following the transfection. Moreover, the MTT assay was performed to identify the cytotoxic effects of doxorubicin and siANRIL alone or in combination on KATO III cells. The effects of si-ANRIL/doxorubicin on KATO III cells migration and apoptosis were assessed by wound healing assay and ELISA cell death method, respectively.

Results: The results showed that the si-ANRIL significantly diminished ANRIL expression level in a time-dependent manner contributing to the distinct repression of cell growth and enhanced apoptosis. Furthermore, the si-ANRIL synergistically elevated the cytotoxic impacts of doxorubicin. Additionally, the ANRIL down-regulation dramatically promoted its induction of apoptosis. Moreover, KATO III cells transfected with si-ANRIL and exposed with doxorubicin revealed significantly reduced invasion capability and enhanced apoptosis rate.

Conclusion: These results demonstrated that the knock-down of IncRNA ANRIL could be a potential therapeutic strategy to trigger apoptosis and circumvent doxorubicin-resistance.
\end{abstract}

Keywords: ANRIL, Apoptosis, Doxorubicin, Drug resistance, Gastric cancer

\section{Background}

Gastric cancer is considered one of the most frequent human malignancies with an increasing rate of incidence worldwide (1). Diagnosis in late advanced stages and malignant proliferation are two important issues increasing the need for focusing more on gastric cancer biology (2). Despite huge advances in the development of novel therapeutic strategies for combating gastric cancer, chemotherapy is still the gold standard strategy for patients with this malignancy (3). However, similar to other cancer types, the development of drug resistance against conventional chemotherapeutic agents is a big burden against the successful treatment of gastric cancer (4). Upregulation in drug efflux pumps, disruption in drug metabolism, increased apoptosis, cancer stem cells, dysregulated DNA repair machinery, and epithelial to mesenchymal transition are among important molecular mechanisms underlying the development of drug resistance (5). Recently, an accumulating number of recent studies has demonstrated the critical roles of long non-coding RNAs (lncRNAs) in various aspects of gastric cancer biology, including drug resistance $(6,7)$. The lncRNAs are a big family of non-coding RNAs with a length size of more than 200 nucleotides, which are not translated to functional proteins; however, they have a major regulatory function in a broad range of biological events (8). Previous studies have explained a long list of various lncRNAs functioning in gastric tumorigenesis and gastric cancer initiation/progression; moreover, they introduced these RNA molecules as potential valuable diagnostic and therapeutic targets for gastric cancer (9). Antisense non-coding RNA in the INK4 locus (ANRIL) situated on the chromosome 9p21 is a wellknown lncRNA with an upregulated expression pattern in gastric cancer $(10,11)$. It was reported that ANRIL overexpression has a strong association with higher tumor stage and size in patients with gastric cancer. In addition, the ANRIL knockdown in both in vitro and pre-clinical studies revealed a decreased proliferation rate of gastric cancer cells (12). Additionally, ANRIL IncRNA has been demonstrated to be implicated in the expansion of drug resistance against cisplatin and 5-fluorouracil in gastric cancer cells (13). However, the role of ANRIL has not been investigated in the development of drug resistance against doxorubicin, which is one of the important chemotherapeutic agents applied in the treatment of gastric cancer.

\section{Objectives}

The current study aimed to explore the effects of silencing ANRIL IncRNA on the proliferation, 
apoptosis, invasion, and drug resistance to doxorubicin in KATO III gastric cancer cells.

\section{Methods}

\subsection{Materials}

The KATO III cell line was provided from Pasteur Institute (Tehran, Iran), and the DOX was obtained from Cayman Chemical (USA). Trypan blue, dimethysulfoxide (DMSO), and 3-(4, 5-dimethylthiazol2-yl)-2,5-diphenyltetrazolium bromide (MTT) were purchased from Sigma Aldrich. Moreover, the RNA extraction kit, cDNA synthesis kit, and SYBR Green were purchased from Bioneer co. (South Korea). Cell culture materials including Trypsin/EDTA, penicillin/ streptomycin (Pen/Strep) solution, fetal bovine serum (FBS), and RPMI-1640 were provided from Invitrogen-Gibco (USA).

\subsection{Cell culture and transfection}

The KATO III cells were grown in RPMI-1640 culture medium supplemented with $10 \%$ heatinactivated FBS and $100 \mathrm{U} / \mathrm{mL}$ antibiotic solution (Pen/Strep). The cells were then maintained at $37^{\circ} \mathrm{C}$ with $5 \% \mathrm{CO}_{2}$, and after reaching $90 \%$ confluency, the KATO III cells were transfected with scrambled negative controls (si-NC) and ANRIL siRNA (siANRIL) by Lipofectamine ${ }^{\text {TM }} 2000$ reagent in order to suppress the expression of ANRIL in cells. Table 1 tabulates the si-ANRIL and si-NC sequences.

\subsection{Cell proliferation assay}

Trypan blue exclusion assay was employed to assess the anti-proliferative effects of si-ANRIL and si-NC on KATO III cells. The viable cells were counted by hemocytometer under the light microscope by conducting the trypan blue dye exclusion experiment.

\subsection{MTT assay}

The viability of KATO III cells was evaluated by MTT assay, and the cultured cells were assigned into three groups of DOX (cells were exposed to different concentrations of DOX [0-4 $\mu \mathrm{M}]$ ), DOX+siNC (cells were exposed to DOX plus si-NC), and DOX+si-ANRIL (cells were exposed to DOX plus siANRIL). After $48 \mathrm{~h}$, the medium was replaced by fresh medium comprising $5 \mathrm{mg} / \mathrm{mL}\left(\begin{array}{ll}20 & \mu \mathrm{l}\end{array}\right)$ MTT solution and maintained for other $4 \mathrm{~h}$. Subsequently, $150 \mu$ l DMSO was used for dissolving the formed crystals of formazan. The absorbance of triplicate experiments was detected at $490 \mathrm{~nm}$. In addition, the synergistic inhibitory impact of DOX and si-ANRIL combination was assessed by the coefficient of drug interaction (CDI) using the following subsequent formula:

$\mathrm{CDI}=\mathrm{AB} /(\mathrm{A} \times \mathrm{B})$, where $\mathrm{A}$ and $\mathrm{B}$ are the ratio of DOX and si-ANRIL to the control group, respectively, and $\mathrm{AB}$ signifies the relative of the co-treatment groups to the control group.

\subsection{RNA extraction and real-time $R T-q P C R$}

Total cellular RNA $(1.5 \mu \mathrm{g})$ of all groups was extracted from KATO III gastric cancer cells with Bioneer specific RNA extraction kit. After evaluating the quality and quantity of RNA samples, the RNAs were subjected to reverse transcription to cDNA using Bioneer kit following the manufacturer's protocols. The Q-PCR was applied using the SYBR Green and special primers on Rotor-Gene ${ }^{\mathrm{TM}} 6000$ system (Corbett Life Science, Australia). The primer sequence for ANRIL was as follow: (Fw) 5'AGGGGGTTGAAATGTGGGTG-3', (Rv) 5'-CTTGAAAG TGGAGAAATAAAGTGCC-3'.

\subsection{Cell apoptosis evaluation}

The apoptosis in KATO III gastric cancer cells and cell death detection ELISA kit was used in order to evaluate the impacts of DOX and si-ANRIL (single or in combination). Following that, a density of $1 \times 10^{4}$ cells per well was plated in 96-well microplates. Subsequently, the cells were exposed to si-ANRIL and si-NC for $48 \mathrm{~h}$, and the cell death was evaluated by commercial ELISA kit according to the instructions.

\subsection{Wound healing assay}

Roughly, $2 \times 10^{4}$ cells were plated into 24-well plates and scraped with pipette tips. After washing with phosphate-buffered saline, the cells were treated with DOX, si-ANRIL, and si-NC (alone or in combination); moreover, they were evaluated under a phase-contrast microscope for 24,48 , and $72 \mathrm{~h}$ post-induction of injury. It is worth mentioning that the migrated cells were measured and quantified using the Image J software.

\subsection{Statistical analysis}

The data were analyzed in SPSS software (version 21 ), and the results were shown as mean \pm SD at least in three replicates. The significance of variances between groups was calculated by ANOVA, and a pvalue less than 0.05 was considered statistically significant.

\section{Results}

4.1. Impacts of si-ANRIL on the expression levels of ANRIL IncRNA

The expression levels of ANRIL IncRNA in siRNA transfected cells were evaluated after 24, 48, and 72 $h$ following transfection. The RT-qPCR results showed that the transfection with si-NC exerted no dramatic impact on the expression levels of ANRIL lncRNA in KATO III cells, compared to controls. As shown in Figure 1, the silencing of ANRIL in gastric cancer cells resulted in significant suppression of ANRIL in a time-dependent manner with a more inhibitory potent effect in $72 \mathrm{~h}$ after si-ANRIL transfection, compared to controls $(\mathrm{P}<0.05)$. 


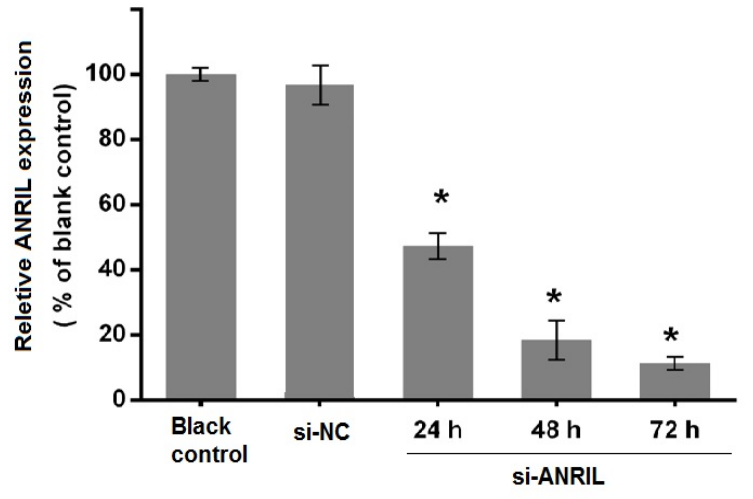

Figure 1. Knock-down of ANRIL lncRNA expression by specific siRNA in KATO III gastric cells

The cells were transfected with si-NC or si-ANRIL for 24, 48, and $72 \mathrm{~h}$. the relative expression of ANRIL was detected using RT-qPCR employing the $2^{-\Delta \Delta \mathrm{C}_{\mathrm{T}}}$ method. The data are depicted as mean $\pm S D(n=4) ; * P<0.05$. si-NC: negative control siRNA; siANRIL: ANRIL siRNA.

\subsection{Anti-proliferative effects of si-ANRIL on KATO III} gastric cancer cells

The cellular viability of KATO III cells following transfection with si-ANRIL and si-NC was evaluated in $1,2,3$, and 4 days. As shown in Figure 2, the transfection with si-NC did not significantly change the viability of gastric cancer cells. However, siANRIL transfection led to significant inhibition of cellular viability in KATO III cells in a time-dependent manner $(\mathrm{P}<0.05)$.

4.3. Potentiating impact of si-ANRIL on the DOXmediated cytotoxic effects in KATO III gastric cancer cells

The impacts of si-ANRIL on the reversing DOXmediated resistant phenotype were assessed by comparing the DOX cytotoxicity in KATO III cells.

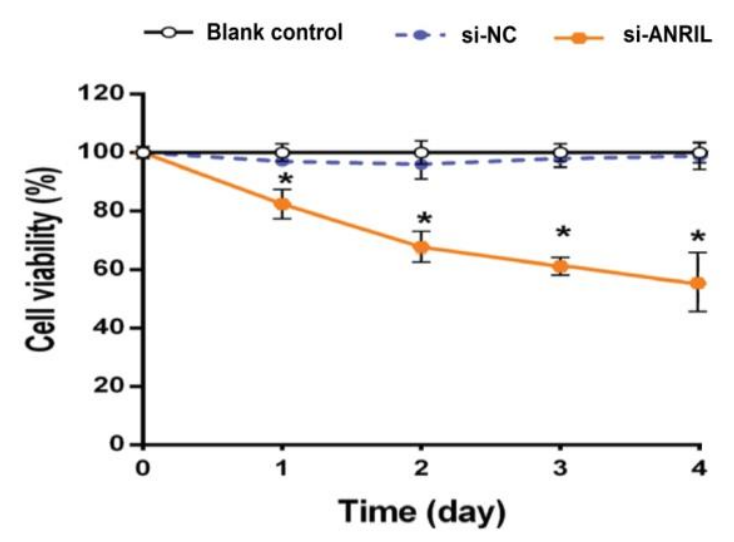

Figure 2. Proliferation repression of KATO III cells exposed to si-NC or si-ARNIL

Cell viability was identified using a trypan blue assay over a period from 0 to 4 days. The data are depicted as mean $\pm S D$ $(\mathrm{n}=5) ;{ }^{*} \mathrm{P}<0.05$ as compared to the blank control. si-NC: negative control siRNA; si-ANRIL: ANRIL siRNA.

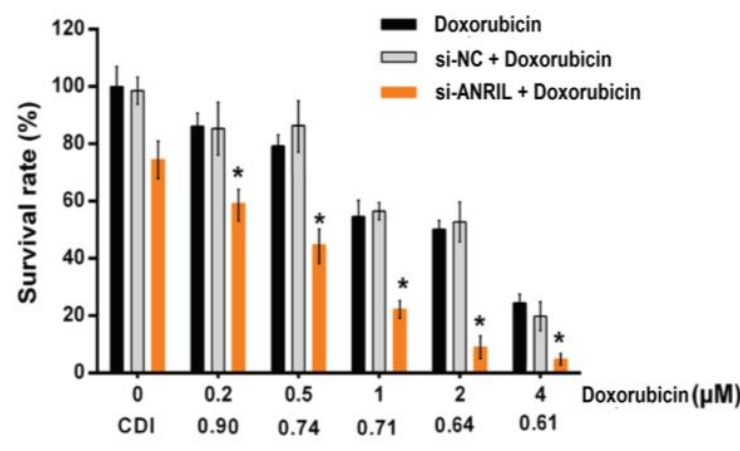

Figure 3. Effect of si-ARNIL/doxorubicin on the DOX-sensitivity of the KATO III cells The KATO III cells were exposed to siARNIL and doxorubicin for $48 \mathrm{~h}$, and their viability was scrutinized using the MTT assay. Moreover, the interaction effect between si-ARNIL and doxorubicin was determined using CDI value. The data are depicted as mean \pm SD $(n=5) ;{ }^{*} \mathrm{P}<0.05$ as compared to doxorubicin or si-ARNIL. si-NC: negative control siRNA; si-ANRIL: ANRIL siRNA; DOX: doxorubicin.

After transfection with si-ANRIL and DOX treatment, the cytotoxicity was evaluated with MTT assay. As presented in Figure 3, DOX-mediated cellular toxicity was dose-dependent. Moreover, the combination treatment of KATO III cells with DOX and si-NC did not exert any significant effects on cellular viability. However, cellular cytotoxicity was significantly higher in cells exposed to the co-treatment of various concentrations of DOX and si-ANRIL $(\mathrm{P}<0.05)$. This suggests that the silencing ANRIL IncRNA in KATO III cells results in the restoration of cancer cell sensitivity to DOX. More importantly, the combined effect of DOX and si-ANRIL was synergistic in KATO III cells as the CDI values were less than 1 at all concentrations (Figure 3).

\subsection{Effects of si-ANRIL on the sensitivity of KATO III cells to DOX-induced apoptosis}

In the next step, it was evaluated whether the combination of DOX and si-ANRIL increased the apoptosis rate of KATO III cells or not. The differences in the apoptosis rate of KATO III cells treated with si-NC and controls were not significant. The cell treatment with DOX or si-ANRIL (alone) resulted in a significant enhancement in apoptosis, compared to the controls $(\mathrm{P}<0.05$; Figure 4). Combination treatment with DOX and si-ANRIL potently enhanced apoptosis in cancer cells vs. cells exposed to DOX or si-ANRIL alone $(\mathrm{P}<0.05)$, which showed that the downregulating ANRIL IncRNA in KAOTIII cells sensitized cells to DOX-induced apoptosis.

\subsection{Effects of si-ANRIL and DOX combination on the KATO III cell migration}

Wound healing techniques were used to investigate the combined effects of DOX si-ANRIL transfection on the migration capability of the KATO 


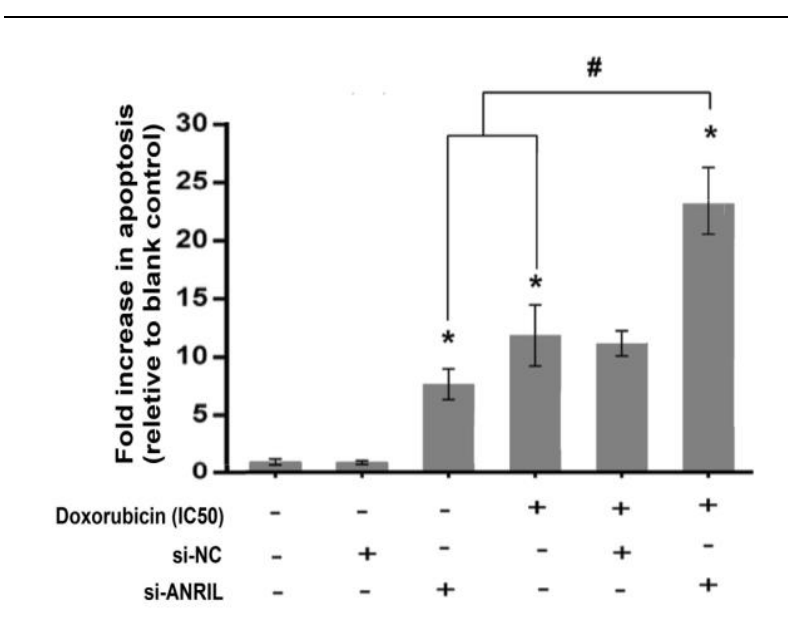

Figure 4. Impact of si-ANRIL on doxorubicin-induced apoptosis The KATO III cell line was treated with doxorubicin in $\mathrm{IC}_{50}$ value $(0.5 \mu \mathrm{M})$ after si-ANRIL transfection. At $48 \mathrm{~h}$ upon the treatment, apoptosis was detected using the ELISA cell death kit. The data are depicted as mean $\pm \mathrm{SD}(\mathrm{n}=4)$; ${ }^{*} \mathrm{P}<0.05$ as compared to the blank control; ${ }^{*} \mathrm{P}<0.05$ vs. doxorubicin or siANRIL alone. si-NC: negative control siRNA; si-ANRIL: ANRIL siRNA; DOX: doxorubicin.

III cells. Accordingly, by comparing the number of cells in the denuded area (Figure 5), it was found that the migration of KATO III cells treated with DOX or siANRIL (alone) was decreased, compared to the control cells after 24,48 , and $72 \mathrm{~h}$ incubation with more potent after $72 \mathrm{~h}(\mathrm{P}<0.05)$. In addition, the combination of DOX and si-ANRIL resulted in a more potent inhibitory effect on the migration of cancer cells at all times $(\mathrm{P}<0.05)$.

\section{Discussion}

The present study demonstrated the efficacy of silencing ANRIL IncRNA in the improvement of the chemo-sensitivity of KATO III cells to DOX. It was found that the co-treatment of cancer cells with DOX and si-ANRIL resulted in a significant inhibition in the cell viability, induction in apoptosis, and suppression of cellular migration. Recent studies have focused on the involvement of various IncRNAs in the different aspects of cancer biology and found that IncRNAs are appropriate candidates for targeted cancer therapy (14-16). Particularly, in gastric cancer, it has been declared that numerous IncRNAs are dysregulated; therefore, they contribute to gastric cancer cellular proliferation, apoptosis, metastasis, angiogenesis, and development of drug resistance to conventional chemotherapeutics $(17,18)$. The DOX is an important chemotherapeutic agent with potent anticancer effects in gastric cancer; however, the development of resistance to this agent strongly limits its efficacy in tumor cells (19). Accordingly, it is of huge importance to understand the molecular mechanism underlying DOX resistance in gastric cancer. Due to the critical role of IncRNAs in gastric cancer initiation/progression, recent studies have

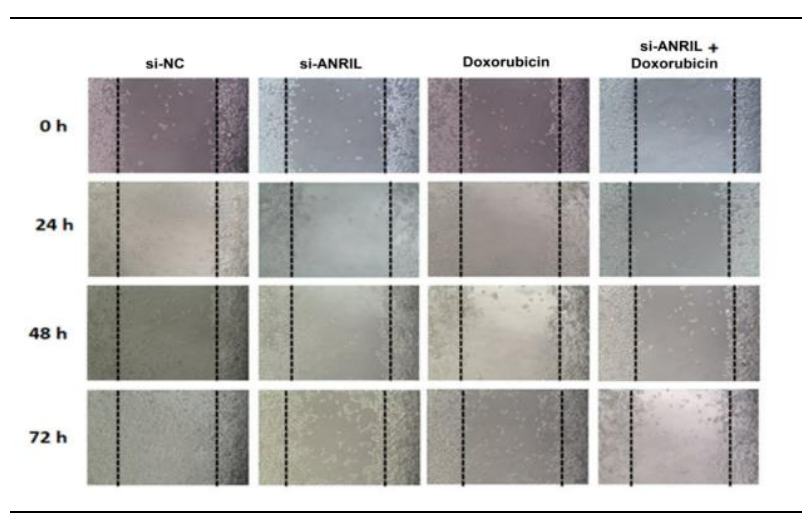

Figure 5. Knockdown of ANRIL inhibits KATO III gastric cancer cell migration Illustrative image of the transfected cells with siNC, si-ANRIL, DOX, and si-ANRIL plus DOX after 24, 48, and 72 h. si-NC: negative control siRNA; si-ANRIL: ANRIL siRNA; DOX: doxorubicin.

examined the involvement of these RNA molecules in the drug resistance in gastric cancer and found interesting results.

In a study by Zhang et al., it was revealed that in the gastric tissues of patients with cisplatin resistance, as well as cisplatin-resistant cancer cells, plasmacytoma variant translocation 1 (PVT-1) lncRNA was overexpressed. Moreover, the upregulation of PVT-1 plays a substantial role in the progression of cisplatinresistance in gastric tumor cells (20). Similarly, Wang et al. illustrated that the expression levels of MDRrelated and upregulated IncRNA (MRUL) was found to be high in DOX-resistant gastric cancer cells, and IncRNA silencing resulted in the induction of apoptosis through increasing DOX accumulation in cancer cells (21). Furthermore, the knockdown of IncRNA adriamycin resistance-associated was shown to increase cancer cells' sensitivity to adriamycin (22). The MEG3 upregulation resulted in a decreased chemosensitivity in cancer cells (23). Therefore, dysregulated lncRNAs have a pivotal function in drug resistance.

The ANRIL as an oncogenic IncRNA was reported to play regulatory roles in cancer biology of various human malignancies, such as lung cancer, hepatocellular carcinoma, as well as gastric and ovarian malignancies (24). Kangarlouei et al. suggested that ANRIL was overexpressed in the tumor tissues of patients with gastric cancer, compared to adjacent normal tissues (25). In the same vein, Deng et al. described that ANRIL IncRNA was upregulated in gastric tumor cells and IncRNA knock-down resulted in elevated apoptosis, hindered tumor progression, and repressed migration of tumor cells (26).

In another study by Liu et al. it was demonstrated that the suppression of ANRIL expression inhibited cell viability, migration, and invasion; however, it increased apoptosis via upregulating miR-99a (27). The results of a study by Lan et al. revealed that the expression levels of 
ANRIL were markedly higher in cisplatin- and 5-FUresistant gastric tumor cells and tissues. More importantly, silencing ANRIL improved cancer cells sensitivity of cisplatin through downregulation of the key drug transporters. Similarly, it was found that silencing ANRIL by a specific siRNA resulted in decreased cellular proliferation and invasion, as well as increased chemosensitivity to DOX in KATO III gastric cancer cells.

\section{Conclusion}

In conclusion, it was found that the knockdown of ANRIL IncRNA led to effective inhibition of KATO III gastric cancer cell proliferation and invasion, as well as increased cancer cells sensitivity to DOX-induced apoptosis. The results of this study suggested that the silencing of IncRNA ANRIL could be a potential therapeutic strategy to trigger apoptosis and circumvent DOX-resistance during chemotherapy. However, further studies are required to explain the exact roles of ANRIL in developing drug resistance in gastric cancer.

\section{Acknowledgements}

The authors would like to thank the Zhongxian Hospital People's Hospital of Chongqing, Chongqing, China.

\section{Footnotes}

Authors' Contribution: Study concept, design, and critical revise: Lina Chen; Acquisition of data: Xuejiang Dai and Hebin Yu; Drafting of the manuscript: Jiahai Che; Statistical analysis: Fei Peng.

Conflict of Interests: None declared.

Ethical Approval: This study was approved by the Ethics Committee of Zhongxian Hospital People's Hospital of Chongqing, Chongqing, China.

Funding/Support: The present study was supported by a grant from Zhongxian Hospital People's Hospital of Chongqing, Chongqing, China.

Informed consent: The informed consent was not required for this study.

\section{References}

1. Sitarz R, Skierucha M, Mielko J, Offerhaus GJA, Maciejewski R, Polkowski WPJCm, et al. Gastric cancer: epidemiology, prevention, classification, and treatment. Cancer Manag Res. 2018;10:239-48. doi: 10.2147/CMAR.S149619. [PubMed: 29445300].

2. Van Cutsem E, Sagaert X, Topal B, Haustermans K, Prenen H. Gastric cancer. Lancet. 2016;388(10060):2654-64. doi: 10.1016/S0140-6736(16)30354-3. [PubMed: 27156933].

3. Wagner AD, Syn NL, Moehler M, Grothe W, Yong WP, Tai BC, et al. Chemotherapy for advanced gastric cancer. 2017(8). doi:10.1002/14651858.CD004064.pub4.

4. Huang T, Song C, Zheng L, Xia L, Li Y, Zhou Y. The roles of extracellular vesicles in gastric cancer development, microenvironment, anti-cancer drug resistance, and therapy.
2019;18(1):62. doi: 10.1186/s12943-019-0967-5. [PubMed: 30925929].

5. Riquelme I, Letelier P, Riffo-Campos AL, Brebi P, Roa JC Emerging role of miRNAs in the drug resistance of gastric cancer. Int J Mol Sci. 2016;17(3):424. doi: 10.3390/ijms 17030424. [PubMed: 27011182].

6. Chen Q-n, Wei C-C, Wang Z-X, Sun M. Long non-coding RNAs in anti-cancer drug resistance. 2017;8(1):1925. doi: 10.18632/ oncotarget.12461. [PubMed: 27713133].

7. Wei L, Sun J, Zhang N, Zheng Y, Wang X, Lv L, et al. Noncoding RNAs in gastric cancer: implications for drug resistance. $\mathrm{Mol}$ Cancer. 2020;19(1):62. doi: 10.1186/s12943-020-01185-7. [PubMed: 32192494].

8. Liz J, Esteller M. IncRNAs and microRNAs with a role in cancer development. Biochim Biophys Acta.2016;1859(1):169-76. doi: 10.1016/j.bbagrm.2015.06.015. [PubMed: 26149773].

9. Hao N-B, He Y-F, Li X-Q, Wang K, Wang R-L. The role of miRNA and lncRNA in gastric cancer. Oncotarget. 2017;8(46):81572. doi: 10.18632/oncotarget.19197. [PubMed: 29113415].

10. Wang J, Sun J, Wang J, Song Y, Gao P, Shi J, et al. Long noncoding RNAs in gastric cancer: functions and clinical applications. Onco Targets Ther. 2016;9:681-97. doi: 10.2147/OTT.S95412. [PubMed: 26929639].

11. Congrains A, Kamide K, Ohishi M, Rakugi H. ANRIL: molecular mechanisms and implications in human health. Int J Mol Sci. 2013;14(1):1278-92. doi: 10.3390/ijms14011278. [PubMed: 23306151].

12. Chen K-H, Chen C-H, Wallace CG, Yuen C-M, Kao G-S, Chen Y-L, et al. Intravenous administration of xenogenic adipose-derived mesenchymal stem cells (ADMSC) and ADMSC-derived exosomes markedly reduced brain infarct volume and preserved neurological function in rat after acute ischemic stroke. Oncotarget. 2016;7(46):74537-56. doi: 10.18632/ oncotarget.12902. [PubMed: 27793019].

13. Lan W-G, Xu D-H, Xu C, Ding C-L, Ning F-L, Zhou Y-L, et al. Silencing of long non-coding RNA ANRIL inhibits the development of multidrug resistance in gastric cancer cells. Oncol Rep 2016;36(1):263-70. doi: 10.3892/or.2016.4771. [PubMed: 27121324].

14. Jiang M-C, Ni J-J, Cui W-Y, Wang B-Y, Zhuo W. Emerging roles of IncRNA in cancer and therapeutic opportunities. Am J Cancer Res. 2019;9(7):1354-66. [PubMed: 31392074].

15. Li J, Meng H, Bai Y, Wang K. Regulation of lncRNA and its role in cancer metastasis. Oncol Res. 2016;23(5):205-17. doi: 10.3727/096504016X14549667334007. [PubMed: 27098144].

16. Evans JR, Feng FY, Chinnaiyan AM. The bright side of dark matter: lncRNAs in cancer. J Clin Invest 2016;126(8):2775-82. doi: 10.1172/JCI84421. [PubMed: 27479746].

17. Gu Y, Chen T, Li G, Yu X, Lu Y, Wang H, et al. LncRNAs: emerging biomarkers in gastric cancer. Future Oncol. 2015;11(17):2427-41. doi: 10.2217/fon.15.175. [PubMed: 26289363]

18. Fang X-Y, Pan H-F, Leng R-X, Ye D-Q. Long noncoding RNAs: novel insights into gastric cancer. Cancer Lett. 2015; 356(2):357-66. doi: 10.1016/j.canlet.2014.11.005. [PubMed: 25444905].

19. Liu X, Lu Y, Xu Y, Hou S, Huang J, Wang B et al. Exosomal transfer of miR-501 confers doxorubicin resistance and tumorigenesis via targeting of BLID in gastric cancer. Cancer Lett. 2019;459:122-34. doi: 10.1016/j.canlet.2019.05.035. [PubMed: 31173853].

20. Zhang X-w, Bu P, Liu L, Zhang X-z, Li J. Overexpression of long non-coding RNA PVT1 in gastric cancer cells promotes the development of multidrug resistance. Biochem Biophys Res Commun. 2015;462(3):227-32. doi: 10.1016/j.bbrc.2015.04.121. [PubMed: 25956062].

21. Wang Y, Zhang D, Wu K, Zhao Q, Nie Y, Fan D. Long noncoding RNA MRUL promotes ABCB1 expression in multidrug-resistant gastric cancer cell sublines. Mol Cell Biol. 2014;34(17):318293. doi: 10.1128/MCB.01580-13. [PubMed: 24958102].

22. Jiang M, Huang O, Xie Z, Wu S, Zhang X, Shen A et al. A novel long non-coding RNA-ARA: adriamycin resistance associated. Biochem Pharmacol 2014;87(2):254-83. doi: 10.1016/j.bcp. 2013.10.020. [PubMed: 24184505]. 
23. Liu J, Wan L, Lu K, Sun M, Pan X, Zhang $P$ et al. The long noncoding RNA MEG3 contributes to cisplatin resistance of human lung adenocarcinoma. PLoS One. 2015;10(5): e0114586. doi: 10.1371/journal.pone.0114586. [PubMed: 25992654].

24. Kong Y, Hsieh C-H, Alonso LC. ANRIL: A IncRNA at the CDKN2A/B Locus with Roles in Cancer and Metabolic Disease. Front Endocrinol (Lausanne).2018;9:405. doi: 10.3389/fendo. 2018.00405. [PubMed: 30087655].

25. Kangarlouei R, Irani S, Noormohammadi Z, Memari F, Mirfakhraie R. ANRIL and ANRASSF1 long noncoding RNAs are upregulated in gastric cancer. J Cell Biochem. 2019; 120(8):12544-8. doi: 10.1002/jcb.28520. [PubMed: 30834580].

26. Deng W, Zhang Y, Cai J, Zhang J, Liu X, Yin J et al. LncRNAANRIL promotes gastric cancer progression by enhancing NFkB signaling. Exp Biol Med (Maywood). 2019;244(12):953-9. doi: 10.1177/1535370219860207. [PubMed: 31242038].

27. Liu P, Zhang M, Niu Q, Zhang F, Yang Y, Jiang X. Knockdown of long non-coding RNA ANRIL inhibits tumorigenesis in human gastric cancer cells via microRNA-99a-mediated downregulation of BMI1. Braz J Med Biol Res. 2018;51(10).e6839. doi: 10.1590/1414-431X20186839. [PubMed: 30156609]. 